\title{
ULTRA-WIDEBAND WAVES THROUGH MULTILAYER PLANAR, CYLINDRICAL AND SPHERICAL MODELS
}

\author{
S. Selim Seker ${ }^{1}$, Isıl Alkocoglu ${ }^{2}$, Fulya Callialp ${ }^{*}{ }^{\boxplus}$ \\ ${ }^{1}$ Department of Electrical and Electronics Engineering, Uskudar University, Istanbul, Turkey \\ 2 Department of Electrical and Electronics Engineering, Bogazici University, Istanbul,Turkey \\ ${ }^{* 3}$ Department of Electrical and Electronics Engineering, Marmara University, Istanbul, Turkey
}

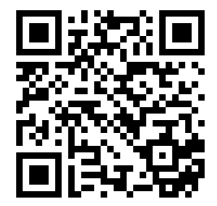

DOI: https://doi.org/10.29121/ijetmr.v7.i7.2020.725

Article Citation: S. Selim Seker, Isıl Alkocoglu, and Fulya Callialp. (2020). ULTRA-WIDEBAND WAVES THROUGH MULTILAYER PLANAR, CYLINDRICAL AND SPHERICAL MODELS. International Journal of Engineering Technologies and Management Research, 7(7), 58-70. https://doi.org/10.29121/ijetmr.v7 i7 2020.725

Published Date: 20 July 2020

Keywords:

Modeling

Multilayer Object

Ultra-Wide Band

\section{ABSTRACT}

This work focuses on modeling part of human body as multilayer planar, cylindrical and spherical shape. Simulations on various human body models using the ultra-wide band are carried out at different incident angles in two scenarios: through wall and without the wall. The absorbed power densities of the layers of a multilayer object are found by taking the material properties and geometric parameters into account. The simulated results are demonstrated and thus compared with the theoretical formulations for the verification. Consequently, the effects of frequency change and wall placement in front of the object are discussed. Our findings indicate that the incident angle of waves effect the reflected and absorbed signal amplitude in the body and with the existence of the wall, the signal amplitude level decrease in the range of $0.1 \%$ and $0.01 \%$ depending on the layer of the body it absorbs.

\section{INTRODUCTION}

Through-the-wall imaging simply makes it possible to determine the layout of a multilayer object and detect what is in it through the outer walls. For a multilayer object, each change in propagation medium induces reflection onto the electromagnetic waves. Then the object of interest can be detected by a proper radar system.

The technology that has low energy levels for transmitting information over a large bandwidth is called UltraWide Band (UWB). UWB technology can share spectrum with other users and transmits information over $500 \mathrm{MHz}$ bandwidths [1]. The difference between traditional radio transmissions and UWB radio transmissions lies in the use of varying the power level, frequency, and phase of a sinusoidal wave. UWB technology is mostly used in location systems as it has low power, high precision capabilities and short broadcasting time [2]. UWB signals have numerous advantages over other signal types when propagating through different objects. Through-the-wall imaging technology is applied for making measurements at some distance without physical contact with the measured object [3]. There are also studies to investigate the feasibility of classifying human activities, human detection and tracking using UWB [4], [5]. Though it has many advantages, the main disadvantage of using UWB signals is the high interference rate. Interference of military UWB radars is still an important problem [6].

This study consists of the adaptation and simulation of UWB through the wall radar and its application for detecting an object of interest. Planar, cylindrical and spherical object modelings are studied, which is essential to model the objects of interest using simpler approaches. The results of different models that are used in this study for

(C) 2020 The Author(s). This is an open access article distributed under the terms of the Creative Commons Attribution License, which permits unrestricted use, distribution, and reproduction in any medium, provided the original author and source are credited. 
the same object (an average human body model) are compared to see the effects of choosing the correct modeling technique.

\section{MATHEMATICAL THEORY}

In order to obtain an expression for the transfer function $\mathrm{H}(\mathrm{j} \omega)$, the electric and magnetic fields of the incident plane-wave, the reflected wave and the transmitted wave are considered. The incident wave is taken as a Gaussian pulse since it is easy to analyze in both time and frequency domain.

The Gaussian pulse is defined with its first and second derivatives as seen in Figure 1 where the standard deviation $\sigma$ is taken as 0.5 :

$$
\begin{aligned}
& f(x)=e^{\frac{-t^{2}}{2 \sigma^{2}}} \\
& f^{\prime}(x)=\frac{t}{\sigma^{2}} f(x) \\
& f^{\prime \prime}(x)=\left(\frac{1}{\sigma^{2}}-\frac{t^{2}}{\sigma^{4}}\right) f(x)
\end{aligned}
$$

\subsection{MULTILAYER PLANAR OBJECTS}

The horizontal ( $\mathbf{E}^{\mathbf{h}}$ ) and vertical ( $\mathbf{E}^{\mathbf{v}}$ ) electric fields inside each layer of Figure 2 can be defined as

$$
\begin{aligned}
& \mathbf{E}_{\mathbf{m}}^{\mathbf{h}}=A_{m} e^{-\gamma_{m} \cdot \mathbf{r}}+B_{m} e^{\gamma_{m} \cdot \mathbf{r}} \\
& \mathbf{E}_{\mathbf{m}}^{\mathbf{v}}=F_{m} e^{-\gamma_{m} \cdot \mathbf{r}}+G_{m} e^{\gamma_{m} \cdot \mathbf{r}}
\end{aligned}
$$
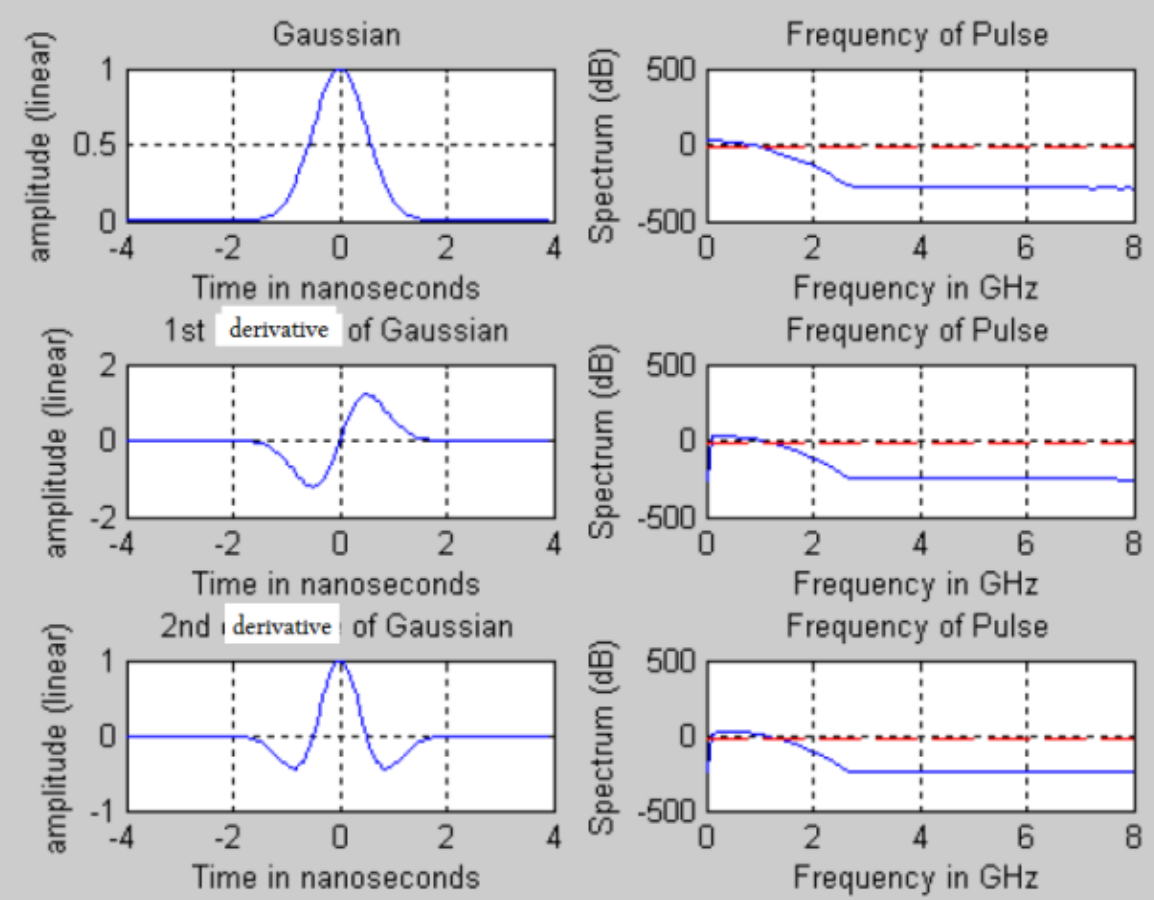

Figure 1: Properties of Gaussian pulse 
S. Selim Seker, Isıl Alkocoglu, and Fulya Callialp

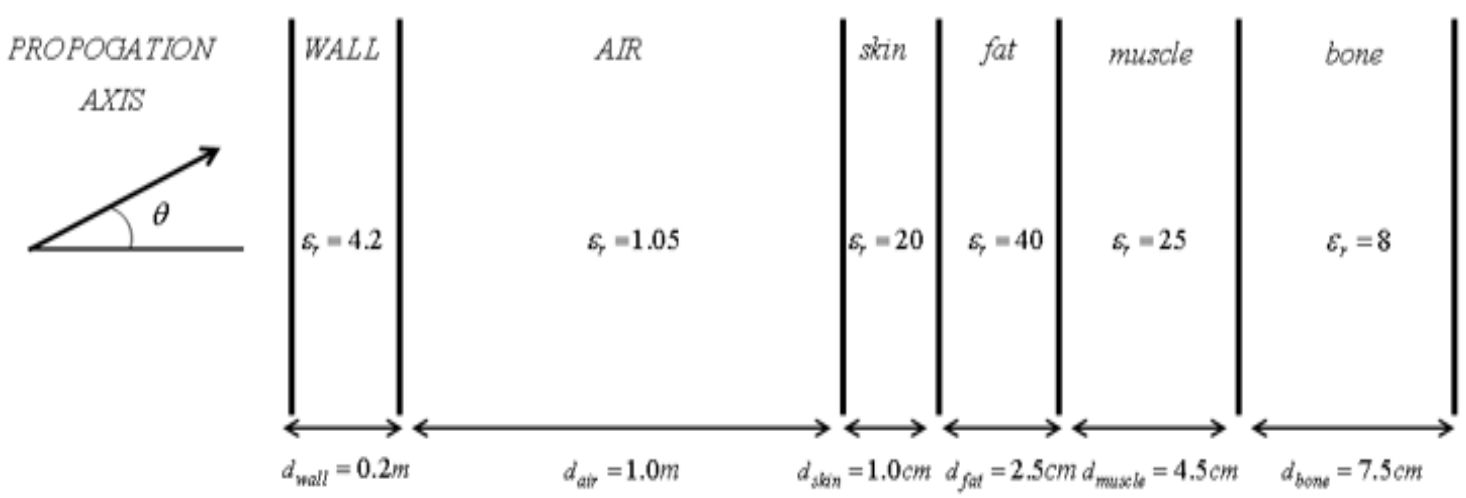

Figure 2: The planar model of the human body

Using boundary conditions, the coefficients for the horizontal and vertical polarized wave, having an incident angle of each layer $\theta_{\mathrm{m}}$ and layer position $\mathrm{d}_{\mathrm{m}}$ upon a multilayer dielectric slab of a relative permittivity $\varepsilon_{\mathrm{m}}$, and the loss tangent $\tan \delta_{\mathrm{m}}$, are [6],

$$
\begin{aligned}
& {\left[\frac{A_{m}}{B_{m}}\right]=\frac{1}{2}\left[\begin{array}{ll}
\left(1+Y_{m+1}\right) e^{\left(\gamma_{m+1}-\gamma_{m}\right) d_{m}} & \left(1-Y_{m+1}\right) e^{-\left(\gamma_{m+1}+\gamma_{m}\right) d_{m}} \\
\left(1-Y_{m+1}\right) e^{\left(\gamma_{m+1}+\gamma_{m}\right) d_{m}} & \left(1+Y_{m+1}\right) e^{\left(\gamma_{m+1}-\gamma_{m}\right) d_{m}}
\end{array}\right]\left[\begin{array}{c}
A_{m+1} \\
B_{m+1}
\end{array}\right]} \\
& {\left[\begin{array}{l}
F_{m} \\
G_{m}
\end{array}\right]=\frac{1}{2}\left[\begin{array}{ll}
\left(1+W_{m+1}\right) e^{\left(\gamma_{m+1}-\gamma_{m}\right) d_{m}} & \left(1-W_{m+1}\right) e^{-\left(\gamma_{m+1}+\gamma_{m}\right) d_{m}} \\
\left(1-W_{m+1}\right) e^{\left(\gamma_{m+1}+\gamma_{m}\right) d_{m}} & \left(1+W_{m+1}\right) e^{\left(\gamma_{m+1}-\gamma_{m}\right) d_{m}}
\end{array}\right]\left[\begin{array}{c}
F_{m+1} \\
G_{m+1}
\end{array}\right]} \\
& Y_{m+1}=\frac{\cos \theta_{m+1}}{\cos \theta_{m} \sqrt{\frac{\varepsilon_{m+1}\left(1-i \tan \delta_{m+1}\right)}{\varepsilon_{m}\left(1-i \tan \delta_{m}\right)}}} \\
& W_{m+1}=\frac{\cos \theta_{m+1}}{\cos \theta_{m} \sqrt{\frac{\varepsilon_{m}\left(1-i \tan \delta_{m}\right)}{\varepsilon_{m+1}\left(1-i \tan \delta_{m+1}\right)}}}
\end{aligned}
$$

The waves pass through a wall and then air before reaching the human body. It is expected for the received signal amplitude to decrease after each layer because of reflection and other loss effects. The electromagnetic fields in each layer are calculated numerically and then the results of planar and cylindrical models are plotted in a graph to compare them with each other.

The transfer function between the incident and transmitted waves of single layer for planar model is:

$$
H(j w)=\frac{4 e^{j \beta_{\mathrm{e}} d}}{e^{\gamma d}\left(2+\frac{\eta_{\mathbf{1}}}{\eta_{\mathbf{2}}}+\frac{\eta_{\mathbf{2}}}{\eta_{\mathbf{1}}}\right)+e^{-\gamma d}\left(2-\frac{\eta_{1}}{\eta_{2}}-\frac{\eta_{2}}{\eta_{1}}\right)}
$$

where $d$ is the thickness of the material, $\beta_{0}$ is the phase constant, and $\eta_{1}=\sqrt{\frac{\mu}{\varepsilon}} \Omega(120 \pi$ in free space $)$ and

$$
\eta_{2}=\sqrt{\frac{\mu}{\varepsilon_{0}\left(\varepsilon_{r}^{\prime}-j \varepsilon_{r}^{\prime \prime}\right)}}=\sqrt{\frac{\mu_{0}}{\varepsilon_{0} \varepsilon_{r}^{\prime}}}=\frac{\eta_{1}}{\sqrt{\varepsilon_{r}^{\prime}}} \quad, \gamma=\alpha+\mathrm{j} \beta=\mathrm{j} \omega \sqrt{\mu \varepsilon\left(\varepsilon^{\prime}-j \varepsilon^{r^{\prime}}\right)}
$$


For a layer of thickness is $0.0014 \mathrm{~m}$ and relative permittivity 4.30 and the incident angle $0, \mathrm{H}(\mathrm{jw})$ is calculated from Eq. (8) as 0.9239.

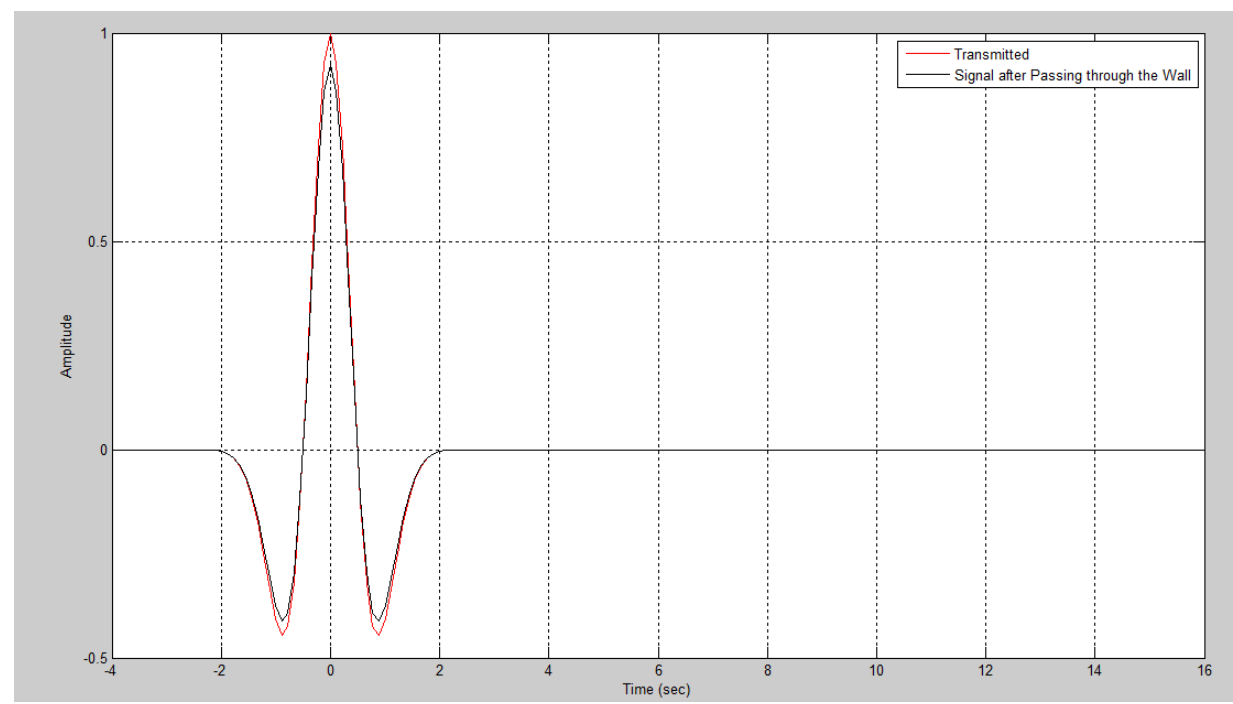

Figure 3: Transmited signal before and after wall for planar model

Figure 3 depicts the verification of the theoretical value and the computed results. It can be seen from Figure 3 that the transmitted wave amplitude is approximately found to be 0.925 , when one layer of thickness is 0.0014 , permittivity is 4.30 at an incident angle of $0^{0}$ degree. It can be concluded that the theoretical value and our result are very close to each other.

\subsection{CYLINDRICAL MULTILAYER OBJECTS}

Assume a plane electromagnetic wave which is incident normally on an infinite long multilayer circular cylinder [7]. Each layer of the multilayer circular cylinder is homogeneous. The usual cylindrical coordinates are employed as illustrated in Figure 4.

The problem is examined in two cases: The TM case with dielectric field $\mathbf{E}_{\mathbf{z}}$ only, and the TE case with the magnetic field $\mathbf{H}_{\mathbf{z}}$ only.

$$
\mathbf{E}_{\mathbf{z}}=\sum_{n=0}^{\infty}\left[e_{n} i^{-n} J_{n}\left(k_{o} r\right)+C_{n} H_{n}^{(2)}\left(k_{o} r\right)\right] \cos n \phi
$$

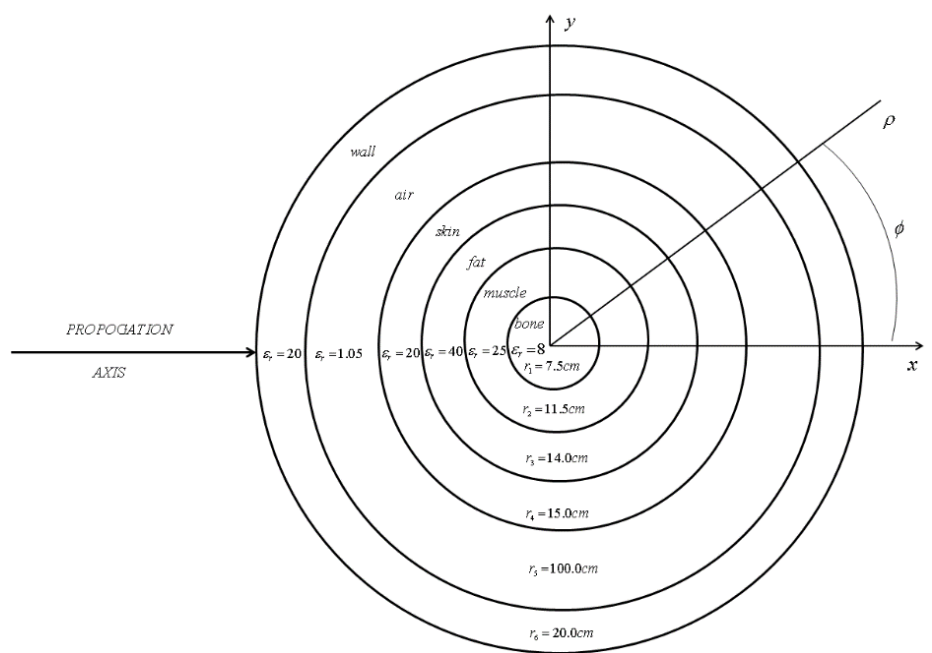

Figure 4: Circular cylindrical model of the object 
In Eq. (10), the first term represents the series expansion for the incident plane wave of unit amplitude.

The fields in the cylindrical space can also be symbolized in terms of the conventional complete set. The successive layers of the cylinder are given numbers of $m=1,2, \ldots, M$ and the outer space is taken as the $(M+1)$ th layer.

The internal field inside the layer $m$ is,

$$
E_{z, m}=\sum_{n=0}^{\infty}\left[A_{m n} J_{n}\left(k_{m} r\right)+B_{m n} Y_{n}\left(k_{m} r\right)\right] \cos n \phi
$$

Where $\mathrm{k}_{\mathrm{m}}=\omega\left(\mu_{\mathrm{m}} \varepsilon_{\mathrm{m}}\right)^{1 / 2}, \mu_{\mathrm{m}}$ and $\varepsilon_{\mathrm{m}}$ are complex material parameters for layer $\mathrm{m}$, and $i=\sqrt{-1}$.

In the first layer, $A_{1, n}^{\prime}=1, B_{1, n}^{\prime}=0$ for all $\mathrm{n}$. The amplitudes are stopped through the layer to the outer space; afterwards they are renormalized to the incident wave by equating the field $E_{z, M+1}$ to the specified field $E_{z}$ of Eq. (10) . The stepping becomes successful by matching $\mathrm{E}_{\mathrm{z}}$ across such cylindrical boundary, where the prime represents the derivative with respect to the argument. The $A^{\prime}, B^{\prime}$ (and also $A, B$ ) amplitudes are obtained by the recursion equation:

$$
\left(\begin{array}{l}
A_{m+1, n}^{\prime} \\
B_{m+1, n}^{\prime}
\end{array}\right)=\left(\begin{array}{ll}
U_{m n} & W_{m n} \\
V_{m n} & X_{m n}
\end{array}\right)\left(\begin{array}{l}
A_{m, n}^{\prime} \\
B_{m, n}^{\prime}
\end{array}\right)
$$

Where

$$
\begin{aligned}
& U_{m n}=\left(\frac{\pi r_{m}}{2 \mu_{m}}\right)\left[\mu_{m} k_{m+1} J_{n}\left(k_{m} r_{m}\right) Y_{n}^{\prime}\left(k_{m+1} r_{m}\right)-\mu_{m+1} k_{m} J_{n}^{\prime}\left(k_{m} r_{m}\right) Y_{n}\left(k_{m+1} r_{m}\right)\right] \\
& V_{m n}=\left(\frac{\pi r_{m}}{2 \mu_{m}}\right)\left[\mu_{m} k_{m+1} J_{n}\left(k_{m+1} r_{m}\right) J_{n}^{\prime}\left(k_{m+1} r_{m}\right)-\mu_{m+1} k_{m} J_{n}^{\prime}\left(k_{m+1} r_{m}\right) J_{n}\left(k_{m} r_{m}\right)\right] \\
& W_{m n}=\left(\frac{\pi r_{m}}{2 \mu_{m}}\right)\left[\mu_{m} k_{m+1} Y_{n}\left(k_{m} r_{m}\right) Y_{n}\left(k_{m+1} r_{m}\right)-\mu_{m+1} k_{m} Y_{n}^{\prime}\left(k_{m} r_{m}\right) Y_{n}\left(k_{m+1} r_{m}\right)\right] \\
& X_{m n}=\left(\frac{\pi r_{m}}{2 \mu_{m}}\right)\left[\mu_{m+1} k_{m} Y_{n}^{\prime}\left(k_{m} r_{m}\right) J_{n}\left(k_{m+1} r_{m}\right)-\mu_{m} k_{m+1} Y_{n}\left(k_{m} r_{m}\right) J_{n}^{\prime}\left(k_{m+1} r_{m}\right)\right]
\end{aligned}
$$

\subsection{MULTILAYER SPHERE OBJECT}

Spherical models have the advantage of taking the body curvatures into account. Therefore, this type of models gives more accurate results than planar multilayer models and especially human head can be represented as a multilayer sphere [8], [9]. Mie Theory is important when the internal and scattered fields of a multilayer sphere are concerned. However, in almost all cases, it is impossible to carry out the exact calculations using the Mie solution. Some approximations and assumptions are made to simplify the task at hand. The multilayer spherical model is preferred as using spherical coordinates provides a symmetry and makes the equation solving process fairly easier [8].

The human head is modeled as a multilayer object, and the model used in this project has six different tissues as layers. The innermost layer represents the brain and the outermost layer represents the skin. These six tissues used in the model have different radii and permittivities. The model consisting of these layers is shown in Figure 5. 
Incident Electric Field:

$E_{i}=E_{0} e^{-i w t} \sum_{n=1}^{\infty} i^{n} \frac{2 n+1}{n(n+1)}\left(m_{01 n}^{(1)}-i n_{e 1 n}^{(1)}\right)$

Reflected Electric Field:

$E_{r}=E_{0} e^{-i w t} \sum_{n=1}^{\infty} i^{n} \frac{2 n+1}{n(n+1)}\left(a_{n}^{r} m_{01 n}^{(3)}-i b_{n}^{r} n_{e 1 n}^{(3)}\right)$

Electric Field Induced within Sphere:

$$
E_{t}=E_{0} e^{-i w t} \sum_{n=1}^{\infty} i^{n} \frac{2 n+1}{n(n+1)}\left(a_{n}^{t} m_{01 n}^{(1)}-i b_{n}^{t} n_{e 1 n}^{(1)}\right)
$$

Scattering Efficiency Factor:

$Q_{s}=\frac{2 \pi}{k_{2}^{2}} \sum_{n=1}^{\infty}(2 n+1)\left(\left|a_{n}^{r}\right|^{2}+\left|b_{n}^{r}\right|^{2}\right)$

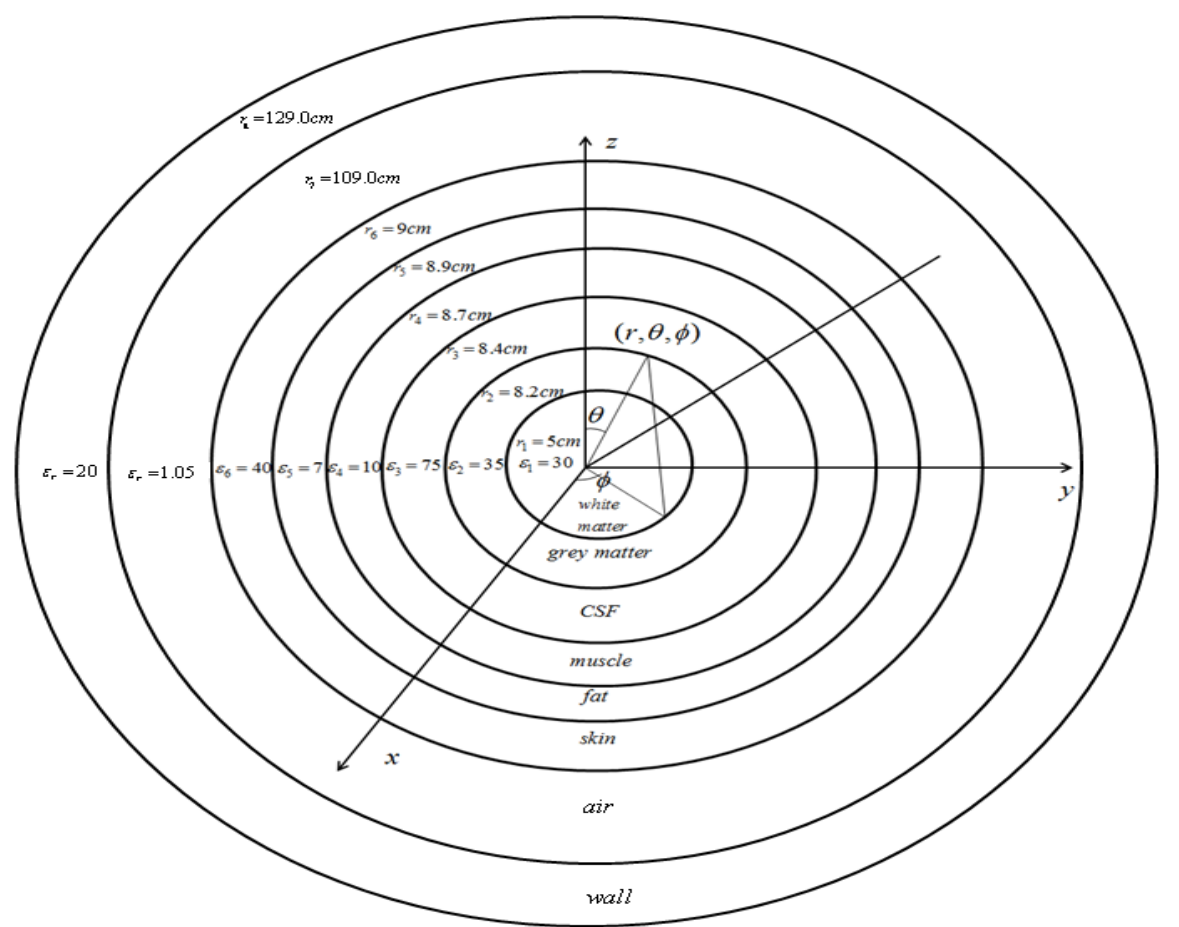

Figure 5: Multilayer spherical human head model with six layers [7]

Total Efficiency Factor:

$Q_{t}=\frac{2 \pi}{k_{2}^{2}} \operatorname{Re} \sum_{n=1}^{\infty}(2 n+1)\left(a_{n}^{r}+b_{n}^{r}\right)$ 
Absorption Efficiency Factor:

$Q_{a}=Q_{t}-Q_{s}$

Where $\mathrm{x}$ is the size parameter, $x=k a=\frac{2 \pi a}{\lambda}$.

The incident wave is assumed as a Gaussian pulse since it is easy to analyze in both time and frequency domain. The incident wave amplitude is normalized and the transmitted wave amplitude after each layer is calculated using the formulas given.

\section{RESULTS AND DISCUSSIONS}

\subsection{THE HUMAN BODY MODEL AS A MULTILAYER PLANAR AND MULTILAYER CYLINDER OBJECT AT 9 GHZ}

In this part of this study, the human body is modeled as a multilayer planar object and multilayer cylinder object at the operating frequency of $9 \mathrm{GHz}$. The used parameters for the human body model are given in the Table 1 [10], [11].

Table 1: Parameters for the human body model

\begin{tabular}{|c|c|c|c|}
\hline & Relative permittivity & Thickness $(\mathrm{cm})$ & Conductivity $(\mathrm{S} / \mathrm{m})$ \\
\hline Wall & 4.2 & 20 & 0.00006 \\
\hline Air & 1.05 & 100 & 0 \\
\hline Skin & 20 & 1.0 & 28 \\
\hline Fat & 40 & 2.5 & 2 \\
\hline Muscle & 25 & 4.0 & 30 \\
\hline Bone & 8 & 7.5 & 7 \\
\hline
\end{tabular}

The average circumference of the human body is assumed to be $94.2 \mathrm{~cm}$ for the cylindrical model while the wall is assumed to be $0.2 \mathrm{~m}$ thick and the air between the body and the wall is $1 \mathrm{~m}$. The simulation for the planar multilayer modeling of the human body and the cylindrical model are given in Figure 6 and in Figure 7, respectively when a $9 \mathrm{GHz}$ normalized amplitude Gaussian pulse is sent at $0^{0}$ incident angle.

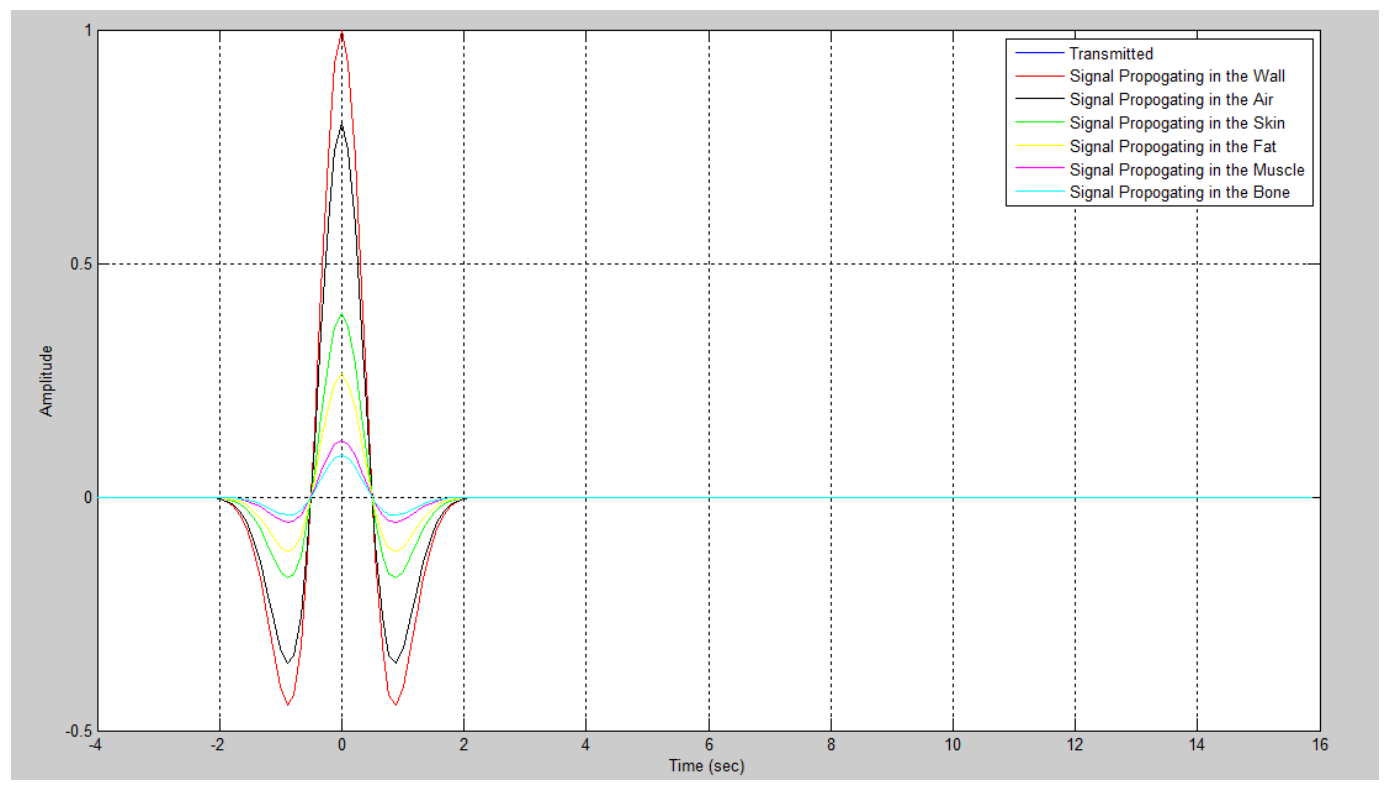

Figure 6: The amplitude of the transmitted wave after each layer of human body according to the multilayer planar model at $0^{\circ}$ incident angle 


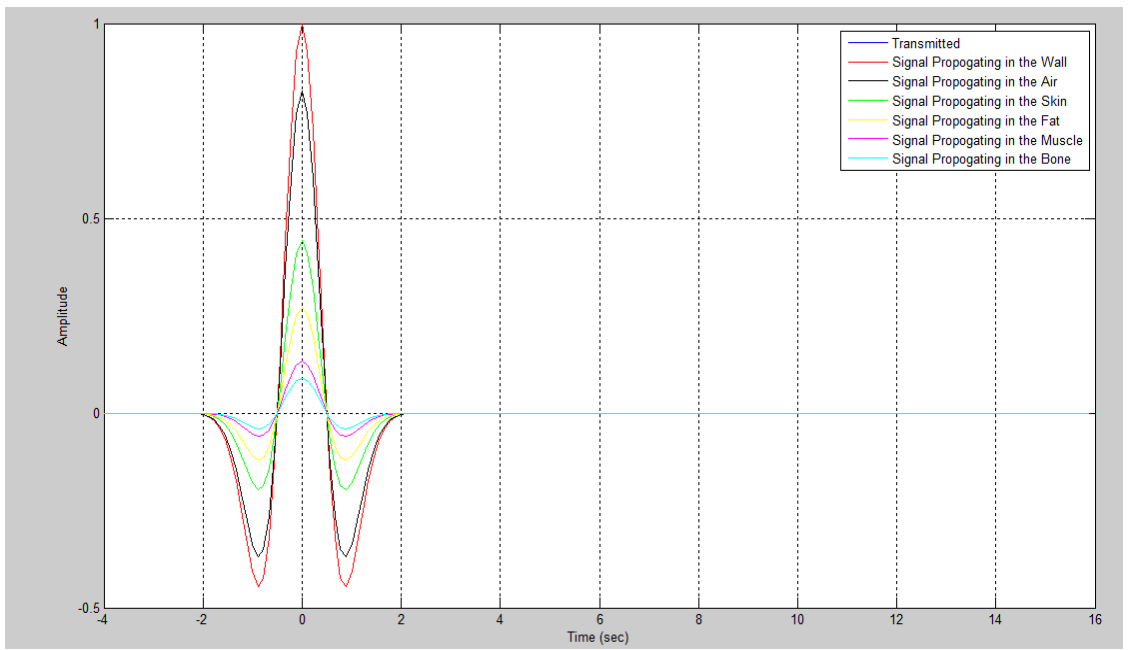

Figure 7: The amplitude of the transmitted wave after each layer of human body according to the multilayer cylindrical model at $0^{\circ}$ incident angle

The simulations are repeated at $60^{\circ}$ incident angle for the planar multilayer modeling and cylindrical multilayer modeling of the human body. The simulations are depicted in Figure 8 and in Figure 9.

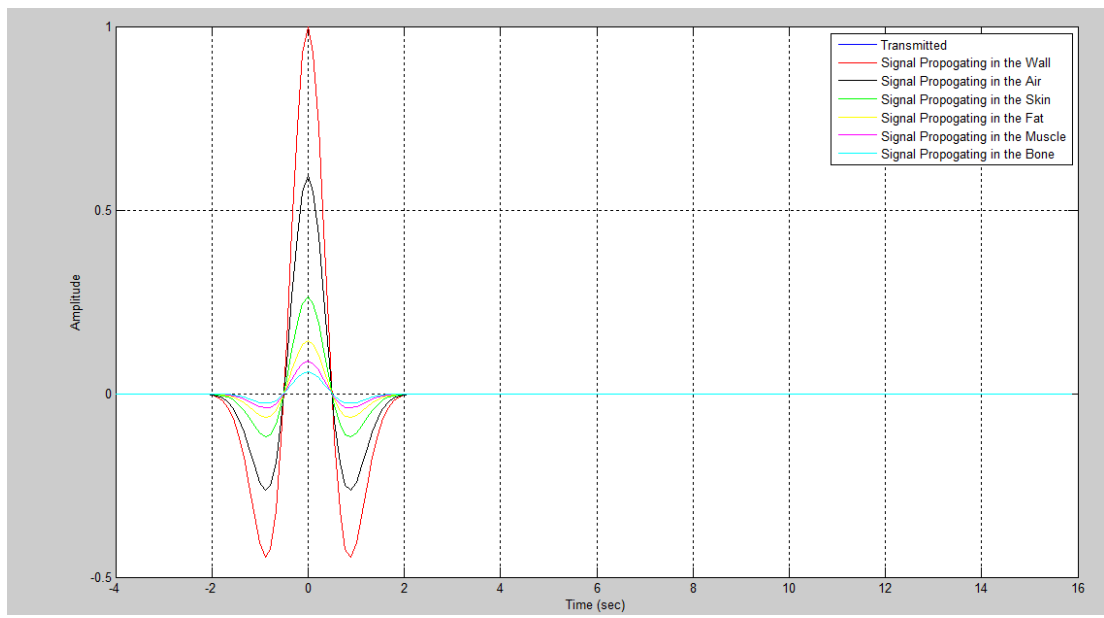

Figure 8: The amplitude of the transmitted wave after each layer of human body according to the multilayer planar model at $60^{\circ}$ incident angle

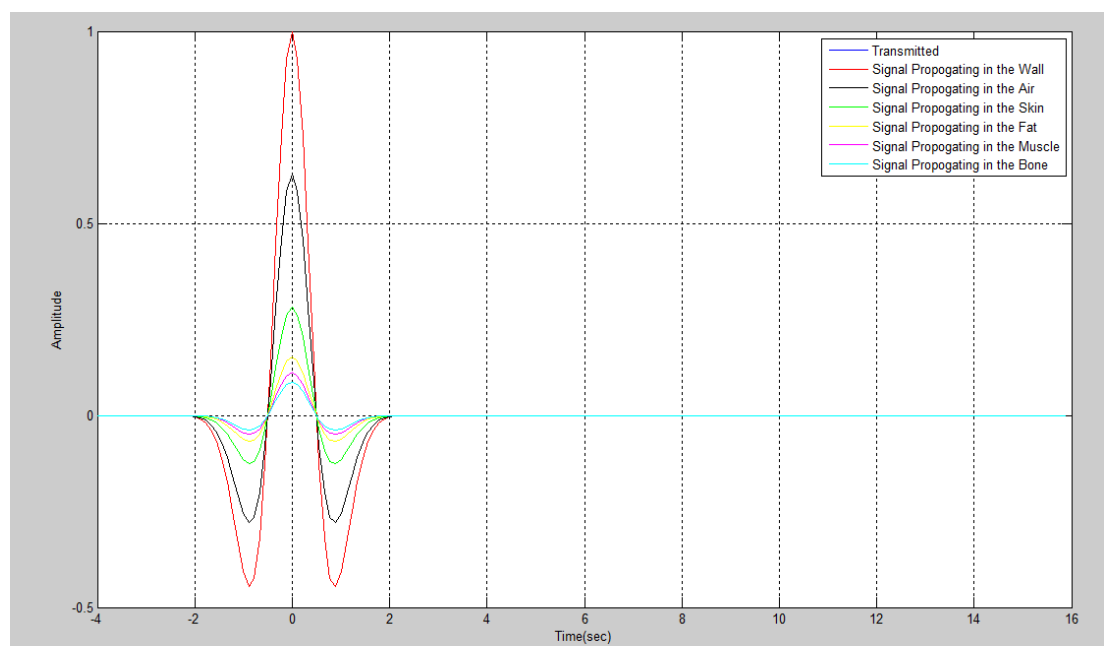

Figure 9: The amplitude of the transmitted wave after each layer of human body according to the multilayer cylindrical model at $60^{\circ}$ incident angle 
In addition, the simulations are done for the nonhuman being case at $9 \mathrm{GHz}$. At $0^{0}$ incident angle, without a human body model behind the wall, the received signal amplitude is measured to be 0.8006 , while after passing through the human, the received amplitude reduced to 0.08843 for the planar model. It is also observed from the findings that, in cylindrical model, the received signal amplitude is 0.8279 , while after passing through the human, the received amplitude reduced to 0.0897 .

At $60^{\circ}$ incidence angle simulations, the received signal amplitude of wave in the planar model is found to be 0.593, while after passing through the human, the received amplitude reduced to 0.05906 . In the cylindrical model it is 0.6287 , while after passing through the human, the received amplitude reduced to 0.0876 . The summarized signal amplitudes values for planar and cylindrical models at $0^{\circ}$ and $60^{\circ}$ incident angles are given in Table 2.

Table 2: The simulated signal amplitudes for planar and cylindrical models at $9 \mathrm{GHz}$

\begin{tabular}{|c|c|c|c|c|}
\hline & $\begin{array}{c}\text { Planar model at } \\
0^{0} \text { incident angle }\end{array}$ & $\begin{array}{c}\text { Cylindrical model at } \\
0^{0} \text { incident angle }\end{array}$ & $\begin{array}{c}\text { Planar model at } \\
0^{0} \text { incident angle }\end{array}$ & $\begin{array}{c}\text { Cylindrical model at } \\
0^{0} \text { incident angle }\end{array}$ \\
\hline $\begin{array}{c}\text { Signal Propagating in } \\
\text { the Wall }\end{array}$ & 1 & 1 & 1 & 0.6287 \\
\hline $\begin{array}{c}\text { Signal Propagating in } \\
\text { the Air }\end{array}$ & 0.8006 & 0.8279 & 0.593 & 0.2814 \\
\hline $\begin{array}{c}\text { Signal Propagating in } \\
\text { the Skin Tissue }\end{array}$ & 0.3918 & 0.4418 & 0.2629 & 0.1523 \\
\hline $\begin{array}{c}\text { Signal Propagating in } \\
\text { the Fat Tissue }\end{array}$ & 0.2621 & 0.2712 & 0.1452 & 0.1112 \\
\hline $\begin{array}{c}\text { Signal Propagating in } \\
\text { the Muscle Tissue }\end{array}$ & 0.121 & 0.1331 & 0.0883 & 0.0876 \\
\hline $\begin{array}{c}\text { Signal Propagating in } \\
\text { the Bone Tissue }\end{array}$ & 0.08843 & 0.0897 & 0.05906 & \\
\hline
\end{tabular}

\subsection{THE HUMAN BODY MODEL AS A MULTILAYER SPHERE OBJECT AT 3 GHZ AND 9 GHZ}

For the multilayer human head model with six layers, the effects of propagation through the layers of human tissue are studied. The received wave amplitudes are plotted for each layer. The waves are considered that they pass through a wall and then air before reaching the human head.

\subsubsection{GHZ FREQUENCY CASE}

The head model parameters at $3 \mathrm{GHz}$ simulations are summarized in Table 3.

Table 3: The human head model layer parameters for $3 \mathrm{GHz}$ [12], [13]

\begin{tabular}{|c|c|c|c|}
\hline & Radius(cm) & Relative Permittivity & Conductivity \\
\hline Wall & 129 & 4.2 & 0.00006 \\
\hline Air & 109 & 1.05 & 0 \\
\hline Skin & 9 & 40 & 30 \\
\hline Fat & 8.9 & 7 & 4 \\
\hline Bone & 8.7 & 10 & 5 \\
\hline CSF & 8.4 & 75 & 0.05 \\
\hline Grey Matter & 8.2 & 35 & 40 \\
\hline White Matter & 5 & 30 & 30 \\
\hline
\end{tabular}

In Figure 10 it is clearly seen that the amplitude of the wave decreases when it passes through the wall. After passing through the air between the wall and the human head, the amplitude is again reduced, but since the air has a low relative permittivity, this decrease is very small. 


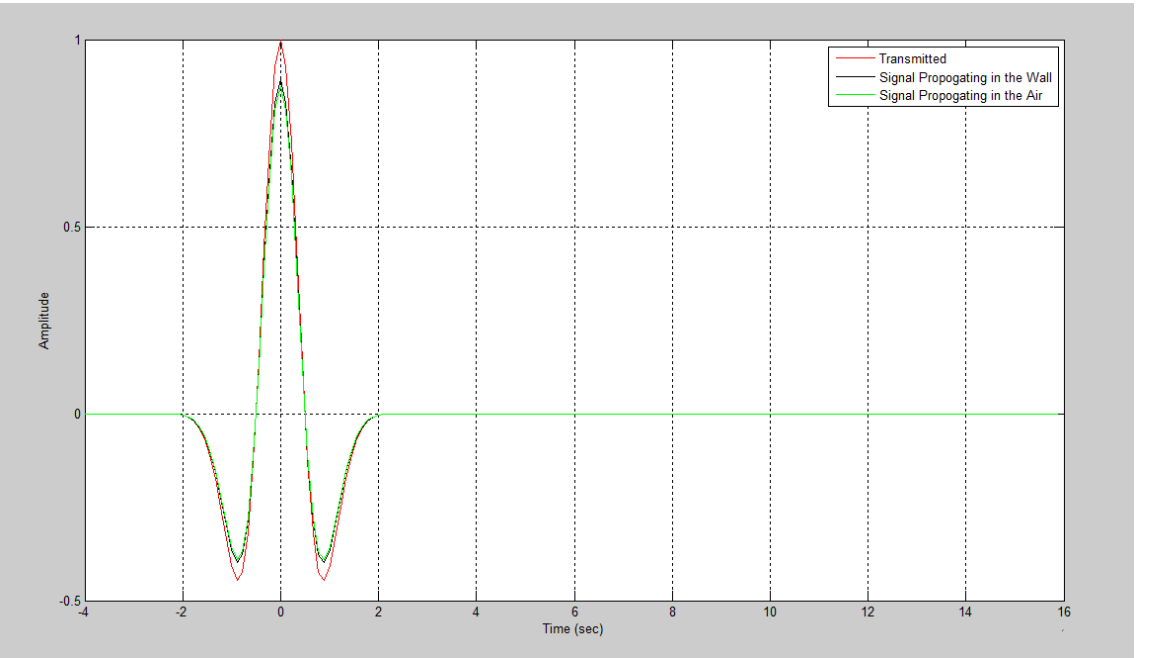

Figure 10: The signal amplitudes after passing through the wall and air at $3 \mathrm{GHz}$

The amplitude received after each layer is plotted in Figure 11. After the air, the waves enter the head and pass through the head tissues in the order of skin, fat, bone, CSF, grey matter and white matter.

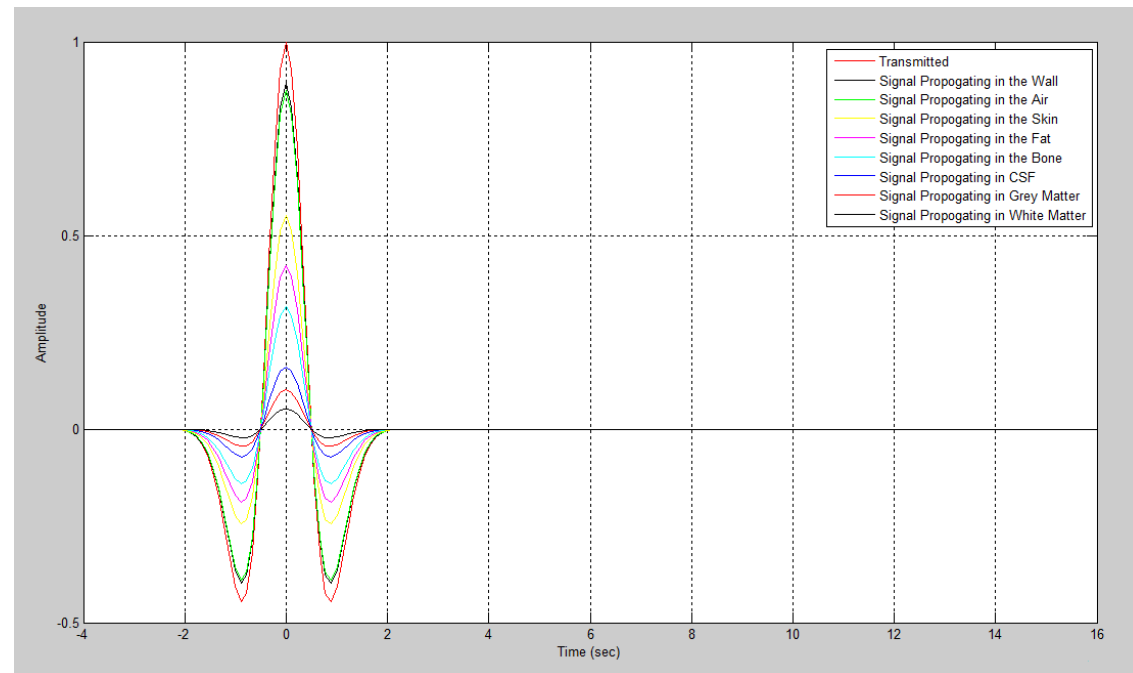

Figure 11: The signal amplitudes after passing through each layer of the human head model at $3 \mathrm{GHz}$

The simulations are also repeated when there is no wall between the electromagnetic wave source and the human head. It is seen that placing a wall between the source and the head reduces the signal amplitude that reaches the outermost layer of the head. When there is no wall placed, the received signal amplitude after the white matter is 0.0689 while it is 0.0520 when a wall is placed due to the propagation loss when passing through the wall. The effect of the wall is summarized in Table where the simulated signal amplitude values are given for all layers in detail.

Table 4: The signal amplitudes with and without the wall at $3 \mathrm{GHz}$

\begin{tabular}{|c|c|c|}
\hline & With the wall & Without the wall \\
\hline Transmitted & 1 & 1 \\
\hline Signal Propagating in The Wall & 0.8933 & - \\
\hline Signal Propagating in The Air & 0.8745 & - \\
\hline Signal Propagating in The Skin & 0.5506 & 0.6297 \\
\hline Signal Propagating in The Fat & 0.4225 & 0.4831 \\
\hline Signal Propagating in The Bone & 0.3161 & 0.3615 \\
\hline Signal Propagating in the CSF & 0.1610 & 0.2134 \\
\hline
\end{tabular}




\begin{tabular}{|c|c|c|}
\hline Signal Propagating in The Grey Matter & 0.1007 & 0.1335 \\
\hline Signal Propagating in The White Matter & 0.0520 & 0.0689 \\
\hline
\end{tabular}

\subsubsection{GHZ FREQUENCY CASE}

At $9 \mathrm{GHz}$ frequency, the signal amplitude variations are both plotted when the signals pass through the wall and air between the wall and when the signals pass through the air and the head tissues including skin, fat, bone, CSF, grey matter and white matter. The findings are shown in Figure 12 and in Figure 13, respectively.

If there is no wall placed between the electromagnetic wave source and the human head, It can be concluded that the results agree with the expectations as the amplitude of the received wave decreases after passing through each layer [2],[13]. Placing a wall between the source and the head again reduces the signal amplitude that reaches the outermost layer of the head. When there is no wall placed, the received signal amplitude after the white matter is 0.0137 while it is 0.0019 for the wall case due to the propagation loss when passing through the wall.

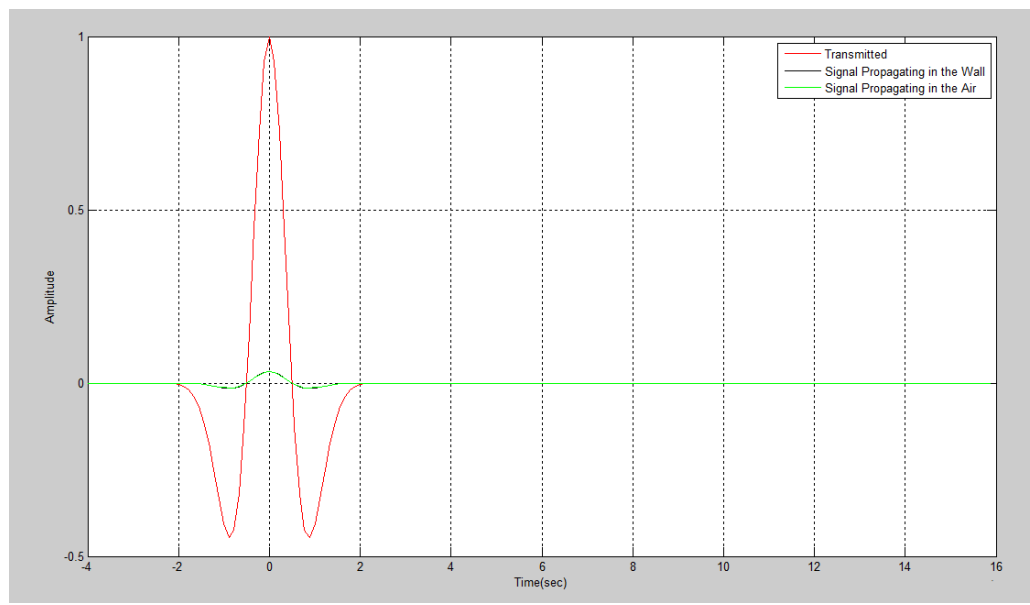

Figure 12: The signal amplitudes after passing through the wall and air at $9 \mathrm{GHz}$

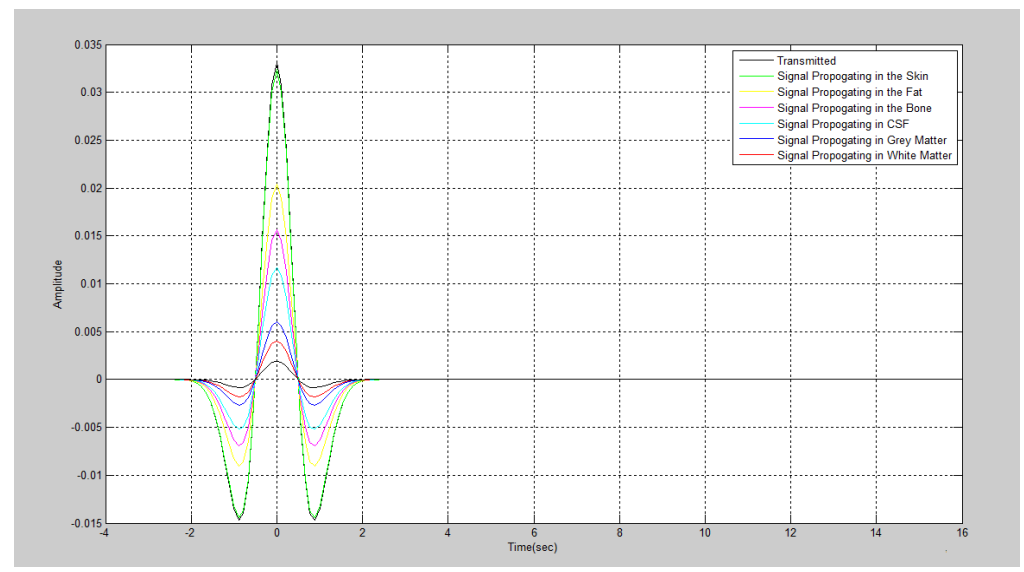

Figure 13: The signal amplitudes after passing through each layer of the human head model for $9 \mathrm{GHz}$.

Table 5: The signal amplitudes with and without the wall at $9 \mathrm{GHz}$

\begin{tabular}{|c|c|c|}
\hline & With the wall & Without the wall \\
\hline Transmitted & 1 & 1 \\
\hline Signal Propagating in The Wall & 0.0007 & - \\
\hline Signal Propagating in The Air & 0.0120 & - \\
\hline Signal Propagating in The Skin & 0.0048 & 0.0319 \\
\hline Signal Propagating in The Fat & 0.0039 & 0.0288 \\
\hline Signal Propagating in The Bone & 0.0057 & 0.0262 \\
\hline
\end{tabular}


Ultra-Wideband Waves Through Multilayer Planar, Cylindrical and Spherical Models

\begin{tabular}{|c|l|l|}
\hline Signal Propagating in the CSF & 0.0020 & 0.0199 \\
\hline Signal Propagating in The Grey Matter & 0.0021 & 0.0150 \\
\hline Signal Propagating in The White Matter & 0.0019 & 0.0137 \\
\hline
\end{tabular}

When the frequency increases from $3 \mathrm{GHz}$ to $9 \mathrm{GHz}$, the transmitted wave amplitude decreases due to reduced skin depth. For the $3 \mathrm{GHz}$ with wall case, $5 \%$ of the incident wave reaches the innermost layer of the brain while for the $9 \mathrm{GHz}$ case this ratio is only $0.2 \%$.

\section{CONCLUSION}

The through-the-wall imaging technology is still developing and it may cause some problems in the long run if seeing through walls clearly is achieved. This may urge people to take some precautions against it, maybe by protecting their places against electromagnetic waves with different mechanisms.

As it is obviously seen from the simulations, two different modeling techniques give similar but not completely matching results. The differences in the received amplitudes are due to different transfer functions in two different geometries. The planar model uses a mathematical transfer function definition for each layer while the cylindrical model has a numerical approach as the mathematical derivation of a transfer function would be very cumbersome. The received amplitudes are close to each other in different multilayer models, giving an expected result.

Since $3 \mathrm{GHz}$ frequency has higher skin depth compared to $9 \mathrm{GHz}$ frequency, the transmitted wave amplitude to the innermost layer (the bone tissue) is higher than $9 \mathrm{GHz}$ case. For $3 \mathrm{GHz}$ frequency and $0^{\circ}$ incident angle for the cylindrical model, the transmitted wave amplitude is 0.0876 , while for the $3 \mathrm{GHz}$ and $0^{\circ}$ incident angle for the cylindrical model, the transmitted wave amplitude is 0.1129 . It can be clearly seen from the $9 \mathrm{GHz}$ and $3 \mathrm{GHz}$ cases for every incident angle that the transmitted wave amplitude is higher for $3 \mathrm{GHz}$ frequency for both planar and cylindrical models.

The suitable parameters for modeling the human head as a multilayer sphere are found. Using electromagnetic characteristics of these four tissues such as permittivity, permeability and conductivity, the wave propagation characteristics of human head are studied. The Mie theory is applied to the six-layer head model for the purposes of through-the-wall human detection and the results are obtained. There are two cases for the incident field: $3 \mathrm{GHz}$ frequency and $9 \mathrm{GHz}$ frequency. The differences arising from the frequency increase is explained and also the effects of placing a wall in front of the head are studied.

\section{SOURCES OF FUNDING}

None.

\section{CONFLICT OF INTEREST}

None.

\section{ACKNOWLEDGMENT}

This work is supported by Bogaziçi University Research Foundation. Project Code 5698.

\section{REFERENCES}

[1] United States. DARPA NETEX program. Ultra-wideband channel propagation measurements and channel modeling. Through-the-Wall Propagation and Material Characterization. Blacksburg, CA, 2002.

[2] Yarovoy, A.G., Lighthart, L.P., Matuzas, J., and Levitas B., UWB Radar for human detection, IEEE A\&E Systems Magazine, March 2006, 10-14.

[3] Kumar, A., Li, Z., Liang, Q., Zhang, B. and X. Wu, Experimental study of through-wall human detection using ultra-wideband radar sensors, Measurement, Vol. 47, January 2014, 869-79. 
[4] Bryan, J.D., Kwon, J., Lee, N. and Y. Kim, Application of ultra-wide band radar for classification of human activities, IET Radar, Sonar \& Navigation, Vol. 6, Issue 3, March 2012, 172-9.

[5] Chang, S., Wolf, M. and J.W. Burdick, Human detection and tracking via ultra-wide band radar, 2010 IEEE International Conference on Robotics and Automation, 3-7 May 2010, Anchorage, AK, USA.

[6] Paris, T.D. and Hurd F.K., Basic Electromagnetic Theory, McGraw-Hill Book Co., New York, 1969.

[7] Bussey, E. H. and J. H. Richmond, Scattering by a Lossy Dielectric Circular Cylindrical Multilayer, Numerical Values, IEEE Transactions on Antennas and Propagation, Vol. 23, 1975, 723-725.

[8] Weil C.M., Absorption Characteristics of Multilayered Sphere Models Exposed UHF/Microwave Radiation, IEEE Transactions, Vol.22, No.6, 1994.

[9] Stratton J.A., Electromagnetic Theory, McGraw Hill New York, 1941.

[10] SSeker, S. and Abatay., H. New frequency-dependent parametric modeling of dielectric materials, Internationsl Journal of Electronic and Communication (AEU) 60, 2006, 320-327.

[11] Eugene S. A. M. and Tijhnis A.G., Pulsed EM Fields in biological tissue, IEEE Engineering in Medicine and Biology Society, Amsterdam, 1996, 1881-82.

[12] Gabriel S., Lau R. W., Gabriel C., The Dielectric Properties of Biological Tissues: III. Parametric Models for the Dielectric Spectrum of Tissues, Physics in Medicine and Biology, 1996.

[13] Lepelaars E.S.A.M, Pulsed EM Fields in Biological Tissue, IEEE Engineering in Medicine and Biology Society, Amsterdam, 1996. 